\section{Hohe Fehlerquote bei der Überprüfung von Apps heimischer Anbieter}

Unter dem Titel "GPEN Privacy Sweep 2014" hat die kanadische Datenschutzaufsichtsbehörde eine Aktion zur Prüfung des Datenschutzes bei Smartphone Apps angestoßen und koordiniert. GPEN steht für "Global Privacy Enforcement Network" und ist ein informeller Zusammenschluss von Datenschutzaufsichtsbehörden auf der ganzen Welt.

In der Woche vom 12. bis 18. Mai 2014 wurden Apps auf Einhaltung der Datenschutzbestimmungen, die in den jeweiligen Teilnehmerländern gelten, überprüft. Auch der Landesbeauftragte für den Datenschutz Baden-Württemberg hat sich an der Aktion beteiligt und vornehmlich Apps evaluiert, die in Baden-Württemberg entwickelt wurden oder deren Betreiber hier ansässig sind.

Dabei wurde festgestellt, dass die meisten Apps die notwendige Transparenz im Umgang mit personenbezogenen Daten vermissen ließen. Hauptmanko war das Fehlen einer Datenschutzerklärung, aus der hervorgeht, warum bestimmte Daten von einer App benötigt werden. Daher konnte auch nicht nachvollzogen werden, ob die erhobenen Daten für das Funktionieren einer App tatsächlich erforderlich waren.

Der Landesbeauftragte ermutigt Besitzer von mobilen Endgeräten wie Smartphones oder Tablets, stets kritisch zu prüfen, welche Zugriffsberechtigungen auf Daten sich eine App einräumt: „Achten Sie darauf, dass eine App nur die Daten anfordert, die für den Anwendungszweck nötig und plausibel sind. So ist Skepsis angebracht, wenn etwa eine Taschenrechner-App auf die Standortdaten oder gar Kontaktdaten zugreifen will. Allzu wissbegierige Anwendungen sollten am besten gar nicht erst installiert oder gleich wieder gelöscht werden“, riet Jörg Klingbeil und kündigte an: „Wir wollen in Zukunft derartige Kontrollaktionen gerne wiederholen, müssen uns dafür allerdings personell noch aufrüsten, denn der Aufwand ist doch beträchtlich."

\section{Methodenhandbuch zum V-Modell XT}

Der IT-Dienstleister und Branchenspezialist msg und die TÜV Informationstechnik GmbH, TÜV NORD GROUP (TÜViT), haben gemeinsam ein Methodenhandbuch zur Durchführung von IT-Projekten für die Bundesanstalt für IT-Dienstleistungen (DLZ-IT) entwickelt. Damit liegt erstmals eine zertifizierte Prozessbeschreibung vor die die Anwendung des V-Modell XT auf IT-Projekte der öffentlichen Verwaltung vereinfacht. Der Auftrag ist Teil eines Rahmenvertrags über Unterstützung beim Projekt-, Anforderungs- und Testmanagement, den msg und TÜViT im vergangenen Jahr mit dem Bundesministerium für Verkehr und digitale Infrastruktur (BMVI) abgeschlossen haben.

\section{Vereinfachte Umsetzung von IT-Projekten}

Das V-Modell XT ist das Standard-Vorgehensmodell für IT-Projekte der öffentlichen Verwaltung. Das Methodenhandbuch des DLZIT vereinfacht die Durchführung derartiger Projekte, indem es ein generisches Phasenmodell, direkt nutzbare Dokumentenvorlagen, Best-Practice-Beispiele sowie konkrete Beschreibungen aller Prozesse von der Projektidee bis zur Inbetriebnahme bereitstellt. Der Schwerpunkt liegt dabei auf auftraggeberseitigen Prozessen wie Projektplanung und -steuerung sowie Anforderungs-, Test- und Lieferantenmanagement. Das Handbuch kann von allen Einheiten im öffentlichen Sektor genutzt werden. Es berücksichtigt die Konzepte des

V-Modell XT, lässt sich aber auch auf andere Projekte übertragen, beispielsweise auf Individualentwicklungen oder SAP-Projekte.

Der Verein WEIT e.V., der die Weiterentwicklung und Pflege des V-Modell XT koordiniert, hat als zuständige Zertifizierungsinstanz die Konformität des Methodenhandbuchs mit dem V-Modell XT bestätigt.

„Das neu entwickelte Methodenhandbuch trägt dazu bei, künftig die Anwendung des V-Modell XT auf unsere IT-Projekte einfacher zu gestalten“, erläutert Dr. Mike Rösler, Projektleiter PMO beim DLZ-IT.

„Wir freuen uns, dass wir der öffentlichen Verwaltung mit dem Handbuch einen leicht nachvollziehbaren methodischen Rahmen zur Durchführung von IT-Projekten zur Verfügung stellen können“, erklärt Jürgen Fritsche, Geschäftsbereichsleiter Public Sector bei msg. „Das Methodenhandbuch ermöglicht die Berücksichtigung organisationsspezifischer Anforderungen. Die konkrete Beschreibung der wichtigsten Prozesse stellt die V-Modell XT-konforme Ausführung sicher", erklärt Antonius Sommer, Geschäftsführer bei TÜViT.

Mehr über die erfolgreiche Zertifizierung des Projektbearbeitungsprozesses des DLZ-IT erfahren Sie hier:. http://www.dlz-it.de/ DE/Aktuelles/Meldungen/2014-05-21_Zertifizierung_Methodenhandbuch_PMO/Zertifizierung_Methodenhandbuch_PMO.html;jsessionid $=9$ C068E9CB51E3A20B7121C4661F83EAC.live2051? $n n=36258$

\section{IT-Sicherheit im Smart Home}

- Wie gut ist das Smart Home gegen IT-Angriffe gerüstet?

- Wie steht es um Sicherheitslücken bei der Smart Home-Steuerung?

- Sind das vernetzte Haus oder das vernetzte Bürogebäude ein Sicherheitsrisiko?

Der Bundesverband IT-Sicherheit e.V. (TeleTrusT) richtet am 12.11.2014 in Berlin einen Informationstag „IT-Sicherheit im Smart Home" aus.

Komfortabler leben im "Smart Home" - Inzwischen gibt es hierfür eine Reihe von Produkten und IT-basierten Lösungen. Wie steht es um IT-Sicherheit und Datenschutz? Experten aus den Bereichen Industrie, Forschung, IT-Sicherheit und Datenschutz ziehen am 12.11.2014 in Berlin Bilanz und präsentieren aktuelle Lösungsansätze. Die Veranstaltung ist interdisziplinär angelegt.

\section{Geplante Themen sind:}

- Aktuelle und zukünftige Funktionalitäten in der Gebäudeautomation, im Smart Home und notwendige IT-Sicherheitsbetrachtungen;

- Sicherheitsanforderungen im Smart Home;

- Security und Gebäudeautomatisierung;

- RWE Smart Home: IT-Sicherheit beim Kunden vor Ort;

- Standardisierung für das Smart Home;

- Das Smart Home als Datenschutzfall.

Programm und Anmeldung unter: https://www.teletrust.de/ veranstaltungen/smart-home/ 